Proceedings of the Edinburgh Mathematical Society (2007) 50,63-71 (C)

DOI:10.1017/S0013091505000921 Printed in the United Kingdom

\title{
THRESHOLD OF GLOBAL EXISTENCE FOR THE CRITICAL NONLINEAR GROSS-PITAEVSKII EQUATION
}

\author{
GUANGGAN CHEN AND JIAN ZHANG \\ College of Mathematics and Software Science, Sichuan Normal University, \\ Chengdu 610066, People's Republic of China (chenguanggan@hotmail.com)
}

(Received 28 June 2005)

\begin{abstract}
This paper is concerned with the critical nonlinear Gross-Pitaevskii equation, which describes the attractive Bose-Einstein condensate under a magnetic trap. We derive a sharp threshold between the global existence and the blowing-up of the system. Furthermore, we answer the question: how small are the initial data, such that the system has global solutions for the nonlinear critical power $p=1+(4 / N)$ ?
\end{abstract}

Keywords: threshold; global existence; blow-up; critical; Gross-Pitaevskii equation

2000 Mathematics subject classification: Primary 35Q55; 35B35; 35A15

\section{Introduction}

In this paper we consider the nonlinear Gross-Pitaevskii equation

$$
\mathrm{i} \varphi_{t}=-\Delta \varphi+|x|^{2} \varphi-|\varphi|^{p-1} \varphi, \quad \varphi(x, 0)=\varphi_{0} .
$$

Here $\varphi: \mathbb{R}^{N} \times[0, \infty) \rightarrow \mathbb{C}$ is a complex-valued function, $1<p<\infty$ when $N=1,2$ and $1<p<(N+2) /(N-2)$ when $N \geqslant 3$. Equation (1.1) models the Bose-Einstein condensate with attractive interparticle interactions under a magnetic trap $[\mathbf{2}, \mathbf{8}, \mathbf{1 3}, \mathbf{1 9}]$.

Meanwhile, as a class of nonlinear Schrödinger equation with potentials, equation (1.1) has a special mathematical significance. For equation (1.1) with a general real-valued potential function $V(x)$, when $\left|D^{\alpha} V(x)\right|$ is bounded for all $\alpha \geqslant 2$, Fujiwara [9] proved that the smoothness of the time 0 of Schrödinger kernel for potentials of quadratic growth. And Yajima [21] showed that, for superquadratic potentials, the Schrödinger kernel is nowhere $C^{1}$. It is shown in [16] that quadratic potentials are the highest order of potential for local well-posedness of the equation. Then $V(x)=|x|^{2}$ is the critical potential for the local existence of the Cauchy problem.

In the case of equation (1.1), Oh [16] and Cazenave [5] established the local existence of the Cauchy problem in the natural energy space. Zhang [24] proved that, when $p<$ $1+(4 / N)$, global solutions of the Cauchy problem (1.1) exist for any initial data in the energy space. On the other hand, when $p \geqslant 1+(4 / N)$, Cazenave [5], Carles $[\mathbf{3}, \mathbf{4}]$ and 
Zhang [23] showed that the solutions of the Cauchy problem (1.1) blow up in a finite time for some initial data, especially for a class of sufficiently large data; but the solutions of the Cauchy problem (1.1) exist globally for other initial data, especially for a class of sufficiently small data $[\mathbf{3}, \mathbf{4}, \mathbf{2 4}]$. So the problem of finding the sharp threshold between the global solutions and the blowing-up solutions for the Cauchy problem (1.1) arises for $p \geqslant 1+(4 / N)$. Owing to the fact that equation (1.1) plays an important role in Bose-Einstein condensates, this problem has also been pursued strongly from a physics point of view $[\mathbf{2}, 6,8,10,12-14,17-19,22]$.

Chen and Zhang [7] obtained a sharp threshold between the global solutions and the blowing-up solutions of the Cauchy problem (1.1), but this result holds only for the case of supercritical power, $p>1+(4 / N)$. On the other hand, for the critical power, $p=1+(4 / N)$, Zhang [23] obtained a sharp condition of global existence for the Cauchy problem (1.1) that was similar to that in Weinstein [20]. However, the result in [23] does not solve the problem of finding the sharp threshold between the global solutions and the blowing-up solutions, because Zhang's result shows that when the initial values $\varphi_{0}$ are less than $N_{\mathrm{c}}$, only global solutions appear; however, when the initial values $\varphi_{0}$ are greater than or equal to $N_{\mathrm{c}}$, there exist not only blowing-up solutions, but also global solutions such as large standing-wave solutions. Here $N_{\mathrm{c}}$ is a number that depends only on the dimension. Therefore, for the critical power, $p=1+(4 / N)$, the problem of finding the sharp threshold between the global solutions and the blowing-up solutions for the Cauchy problem (1.1) is still open. In this paper, we shall solve this problem.

In this paper we fix $p=1+(4 / N)$. In the next section, we give some preliminaries. In $\S 3$, we construct some proper functionals, and pose a constrained variational problem, which we then solve. In $\S 4$, by combining the variational character with the invariant properties of the local semi-flows of the evolution system, we get a sharp threshold between the global existence and blowing-up. These arguments originate in $[\mathbf{1}, \mathbf{2 5}]$ and in Levine's concavity method $[\mathbf{1 5}]$. Furthermore, we answer the question: how small are the initial data, such that the Cauchy problem (1.1) has global solutions for $p=1+(4 / N)$ ?

\section{Preliminaries}

Firstly, we naturally set

$$
H:=\left\{u \in H^{1}\left(\mathbb{R}^{N}\right): \int|x|^{2}|u|^{2} \mathrm{~d} x<\infty\right\},
$$

where $H^{1}\left(\mathbb{R}^{N}\right)=\left\{u: u \in L^{2}\left(\mathbb{R}^{N}\right)\right.$ and $\left.\partial_{x_{i}} u \in L^{2}\left(\mathbb{R}^{N}\right), i=1,2, \ldots, N\right\}$. Henceforth, for simplicity, we denote $\int_{\mathbb{R}^{N}} \cdot \mathrm{d} x$ by $\int \cdot \mathrm{d} x$. $H$ becomes a Hilbert space, continuously embedded in $H^{1}\left(\mathbb{R}^{N}\right)$, when endowed with the inner product

$$
\langle\varphi, \psi\rangle_{H}=\int\left[\nabla \varphi \nabla \bar{\psi}+\varphi \bar{\psi}+|x|^{2} \varphi \bar{\psi}\right] \mathrm{d} x,
$$

whose associated norm we denote by $\|\cdot\|_{H}$. In addition, we use $\|\cdot\|_{p}$ to denote the norm of $L^{p}\left(\mathbb{R}^{N}\right)$. 
Lemma 2.1 (Zhang [24]). Let $1 \leqslant p<\infty$. Then the embedding $H \hookrightarrow L^{p+1}\left(\mathbb{R}^{N}\right)$ is compact.

Proposition 2.2 (Cazenave [5], Glassey [11], Oh [16]). Assume that $1<p<\infty$ when $N=1,2,1<p<(N+2) /(N-2)$ when $N \geqslant 3$ and $\varphi_{0} \in H$. There then exists a unique solution $\varphi$ of the Cauchy problem $(1.1)$ in $C([0, T) ; H)$ for some $T \in[0, \infty)$ (maximal existence time), and $\varphi$ satisfies the following two mass- and energy-conservation laws:

$$
\begin{aligned}
M(\varphi) & :=\int|\varphi|^{2} \mathrm{~d} x=M\left(\varphi_{0}\right), \\
E(\varphi) & :=\int\left[|\nabla \varphi|^{2}+|x|^{2}|\varphi|^{2}-\frac{2}{p+1}|\varphi|^{p+1}\right] \mathrm{d} x=E\left(\varphi_{0}\right),
\end{aligned}
$$

for all $t \in[0, T)$. Furthermore, we have the following alternatives: $T=\infty$ or else $T<\infty$ and $\lim _{t \rightarrow T}\|\varphi\|_{H}=\infty$ (blow-up).

Proposition 2.3 (Cazenave [5]). Let $\varphi_{0} \in H$. Then for $1<p<1+(4 / N)$, the Cauchy problem (1.1) has a unique bounded global solution $\varphi$ on $t \in[0, \infty)$ in $H$. For $p \geqslant 1+(4 / N)$, when $\left\|\varphi_{0}\right\|_{H}$ is sufficiently small, the Cauchy problem (1.1) has a unique bounded global solution in $H$; when $\left\|\varphi_{0}\right\|_{H}$ is sufficiently large, the Cauchy problem (1.1) has a unique solution blowing up in a finite time in $H$.

Proposition 2.4 (Cazenave [5]). For $p \geqslant 1+(4 / N)$, when $E\left(\varphi_{0}\right)<0$, the solution $\varphi$ of the Cauchy problem (1.1) blows up in a finite time in $H$.

Proposition 2.5 (Cazenave [5]). Let $\varphi_{0} \in H$ and let $\varphi$ be a solution of the Cauchy problem (1.1) on $[0, T)$. Set

$$
J(t)=\int|x|^{2}|\varphi|^{2} \mathrm{~d} x
$$

Then one has

$$
\frac{\mathrm{d}^{2}}{\mathrm{~d} t^{2}} J(t)=8 \int\left[|\nabla \varphi|^{2}-|x|^{2}|\varphi|^{2}-\frac{N(p-1)}{2(p+1)}|\varphi|^{p+1}\right] \mathrm{d} x .
$$

Lemma 2.6 (Weinstein [20]). Let $\varphi \in H$. Then we have

$$
\int|\varphi|^{2} \mathrm{~d} x \leqslant \frac{2}{N}\left(\int|\nabla \varphi|^{2} \mathrm{~d} x\right)^{1 / 2}\left(\int|x|^{2}|\varphi|^{2} \mathrm{~d} x\right)^{1 / 2} .
$$

\section{A variational problem}

We define two functionals on $H$ as follows

$$
S(u)=\frac{1}{2} \int\left[|\nabla u|^{2}+|x|^{2}|u|^{2}\right] \mathrm{d} x
$$

and

$$
R(u)=\frac{1}{2} \int\left[|u|^{2}-\frac{2}{p+1}|u|^{p+1}\right] \mathrm{d} x .
$$


Then set

$$
\left.\begin{array}{rl}
d & =\inf _{u \in \Sigma} S(u), \\
\Sigma & =\{u \in H \backslash\{0\}: R(u)=0\} .
\end{array}\right\}
$$

Theorem 3.1. Let $p=1+(4 / N)$. There then exists a $u \in \Sigma$ such that

$$
d=\min _{u \in \Sigma} S(u)
$$

Furthermore, $d>0$.

Proof. Choose a minimizing sequence $\left\{u_{n}\right\}_{n \in \mathbb{N}}$, so we have $u_{n} \in \Sigma$ and

$$
\lim _{n \rightarrow \infty} \frac{1}{2} \int\left[\left|\nabla u_{n}\right|^{2}+|x|^{2}\left|u_{n}\right|^{2}\right] \mathrm{d} x=d .
$$

By the Gagliardo-Nirenberg inequality and $u_{n} \in \Sigma$, we have

$$
0<\int\left|u_{n}\right|^{2} \mathrm{~d} x=\frac{2}{p+1} \int\left|u_{n}\right|^{p+1} \mathrm{~d} x \leqslant C\left\|\nabla u_{n}\right\|_{2}^{p-1}\left\|u_{n}\right\|_{2}^{2} .
$$

Henceforth, for simplicity, we use $C$ to denote various positive constants. Therefore, there exists a positive constant $C$ such that

$$
\int\left|\nabla u_{n}\right|^{2} \mathrm{~d} x \geqslant C>0
$$

which implies that

$$
\frac{1}{2} \int\left[\left|\nabla u_{n}\right|^{2}+|x|^{2}\left|u_{n}\right|^{2}\right] \mathrm{d} x \geqslant C>0
$$

Hence, $d>0$.

In addition, it follows from (3.5), Lemma 2.6 and the Cauchy inequality that

$$
\begin{aligned}
\int\left|u_{n}\right|^{2} \mathrm{~d} x & \leqslant \frac{2}{N}\left(\int\left|\nabla u_{n}\right|^{2} \mathrm{~d} x\right)^{1 / 2}\left(\int|x|^{2}\left|u_{n}\right|^{2} \mathrm{~d} x\right)^{1 / 2} \\
& \leqslant \frac{1}{N}\left(\int\left|\nabla u_{n}\right|^{2} \mathrm{~d} x+\int|x|^{2}\left|u_{n}\right|^{2} \mathrm{~d} x\right) \\
& <C .
\end{aligned}
$$

By (3.5) and (3.8), one finds that $\left\{u_{n}\right\}_{n \in \mathbb{N}}$ is bounded in $H$. Therefore, there exists $u \in H$ such that a subsequence of $\left\{u_{n}\right\}_{n \in \mathbb{N}}$ (which we still denote by $\left\{u_{n}\right\}_{n \in \mathbb{N}}$ ) satisfies

$$
u_{n} \rightarrow u \quad \text { weakly in } H \text {. }
$$

By Lemma 2.1 we have

$$
\left.\begin{array}{ll}
u_{n} \rightarrow u & \text { in } L^{2}\left(\mathbb{R}^{N}\right), \\
u_{n} \rightarrow u & \text { in } L^{p+1}\left(\mathbb{R}^{N}\right) .
\end{array}\right\}
$$


Hence,

$$
R(u)=\frac{1}{2} \int\left[|u|^{2}-\frac{2}{p+1}|u|^{p+1}\right] \mathrm{d} x=0,
$$

and $u \in \Sigma$, so we have $S(u) \geqslant d$. On the other hand, since $S\left(u_{n}\right)$ is coercive and convex, one has

$$
S(u) \leqslant \liminf _{n \rightarrow \infty} S\left(u_{n}\right) .
$$

Therefore, from (3.5) and (3.12), we have

$$
d \leqslant S(u) \leqslant \liminf _{n \rightarrow \infty} S\left(u_{n}\right) \leqslant \lim _{n \rightarrow \infty} S\left(u_{n}\right)=d
$$

which implies that $S(u)=d$. Therefore, Theorem 3.1 is true.

\section{Threshold of global existence}

In this section, we shall give the main results and the proofs. First, we define another functional in $H$ as

$$
I(u)=\frac{1}{2} \int\left[|\nabla u|^{2}+|u|^{2}+|x|^{2}|u|^{2}-\frac{2}{p+1}|u|^{p+1}\right] \mathrm{d} x .
$$

Hence, we can obtain the invariant properties of the local semi-flows of the Cauchy problem (1.1).

Proposition 4.1. Let $p=1+(4 / N)$ and

$$
\left.\begin{array}{l}
K_{+}=\{u \in H: R(u)>0, I(u)<d\}, \\
K_{-}=\{u \in H: R(u)<0, I(u)<d\} .
\end{array}\right\}
$$

Then $K_{+}$and $K_{-}$are invariant under the local semi-flow generated by the Cauchy problem (1.1).

Proof. Let $\varphi_{0} \in K_{+}$and let $\varphi$ be the unique solution of (1.1) with the initial datum $\varphi_{0}$. Therefore, it follows easily from Proposition 2.2 that $I(\varphi)=\frac{1}{2}[M(\varphi)+E(\varphi)]=$ $\frac{1}{2}\left[M\left(\varphi_{0}\right)+E\left(\varphi_{0}\right)\right]=I\left(\varphi_{0}\right), t \in[0, T)$. Thus, from $I\left(\varphi_{0}\right)<d$, it follows that

$$
I(\varphi)<d, \quad t \in[0, T) .
$$

To check that $\varphi \in K_{+}$, we need to prove that

$$
R(\varphi)>0, \quad t \in[0, T) .
$$

If it were not, because of $R\left(\varphi_{0}\right)>0$, there would exist, by continuity, a $t_{1} \in(0, T)$ such that $R\left(\varphi\left(t_{1}\right)\right)=0$. Then $\varphi\left(t_{1}\right) \in \Sigma$. Hence, $S\left(\varphi\left(t_{1}\right)\right) \geqslant d$. From (3.1), (3.2) and (4.1), $I\left(\varphi\left(t_{1}\right)\right)=S\left(\varphi\left(t_{1}\right)\right)+R\left(\varphi\left(t_{1}\right)\right)$. It follows from (4.3) that $S\left(\varphi\left(t_{1}\right)\right)<d$, violating Theorem 3.1. Therefore, (4.4) holds. Hence, $K_{+}$is invariant under the local semi-flow generated by the Cauchy problem (1.1).

By the same argument as that above, we can show that $K_{-}$is invariant. 
Theorem 4.2. For $p=1+(4 / N)$ and $N \geqslant 2$, let $\varphi_{0} \in H$. We then have the following.

(i) If $\varphi_{0} \in K_{+}$, the solution $\varphi$ of the Cauchy problem (1.1) exists globally on $t \in[0, \infty)$ in $H$.

(ii) If $\varphi_{0} \in K_{-}$, the solution $\varphi$ of the Cauchy problem (1.1) blows up at a finite time in $H$.

Proof. (i) Let $\varphi_{0} \in K_{+}$and let $\varphi$ be the solution of (1.1) with the initial datum $\varphi_{0}$. It follows from Proposition 4.1 that $\varphi \in K_{+}$, i.e.

$$
\left.\begin{array}{rl}
I(\varphi) & =\frac{1}{2} \int\left[|\nabla \varphi|^{2}+|\varphi|^{2}+|x|^{2}|\varphi|^{2}-\frac{2}{p+1}|\varphi|^{p+1}\right] \mathrm{d} x<d, \\
R(\varphi) & =\frac{1}{2} \int\left[|\varphi|^{2}-\frac{2}{p+1}|\varphi|^{p+1}\right] \mathrm{d} x>0,
\end{array}\right\}
$$

which implies that

$$
\frac{1}{2} \int\left[|\nabla \varphi|^{2}+|x|^{2}|\varphi|^{2}\right] \mathrm{d} x
$$

is bounded. Furthermore, since

$$
\int|\varphi|^{2} \mathrm{~d} x=\int\left|\varphi_{0}\right|^{2} \mathrm{~d} x
$$

we then find that $\|\varphi\|_{H}$ is bounded. Therefore, it follows from Proposition 2.2 that the solution $\varphi$ of the Cauchy problem (1.1) exists globally on $t \in[0, \infty)$ in $H$.

(ii) Let $\varphi_{0} \in K_{-}$and let $\varphi$ be the solution of (1.1) with the initial datum $\varphi_{0}$. It follows from Proposition 4.1 that $\varphi \in K_{-}$, i.e.

$$
I(\varphi)=\frac{1}{2} \int\left[|\nabla \varphi|^{2}+|\varphi|^{2}+|x|^{2}|\varphi|^{2}-\frac{2}{p+1}|\varphi|^{p+1}\right] \mathrm{d} x<d,
$$

and

$$
R(\varphi)=\frac{1}{2} \int\left[|\varphi|^{2}-\frac{2}{p+1}|\varphi|^{p+1}\right] \mathrm{d} x<0 .
$$

Therefore, there must exist a $\lambda \in(0,1)$ such that $R(\lambda \varphi)=0$, which implies that $\lambda \varphi \in \Sigma$. So, $S(\lambda \varphi) \geqslant d>I(\varphi)$, i.e.

$$
\lambda^{2} \int\left[|\nabla \varphi|^{2}+|x|^{2}|\varphi|^{2}\right] \mathrm{d} x>\int\left[|\nabla \varphi|^{2}+|\varphi|^{2}+|x|^{2}|\varphi|^{2}-\frac{2}{p+1}|\varphi|^{p+1}\right] \mathrm{d} x
$$

When $N=2$ and $p=3$, it follows from $R(\lambda \varphi)=0$ and (4.8) that

$$
\left(\lambda^{2}-1\right) \int\left[|\nabla \varphi|^{2}+|x|^{2}|\varphi|^{2}-\frac{1}{2}|\varphi|^{4}\right] \mathrm{d} x>0 .
$$


At the same time, noting that $\lambda \in(0,1)$, we have

$$
E(\varphi)=\int\left[|\nabla \varphi|^{2}+|x|^{2}|\varphi|^{2}-\frac{1}{2}|\varphi|^{4}\right] \mathrm{d} x<0 .
$$

It then follows from this result and Proposition 2.2 that $E\left(\varphi_{0}\right)<0$. Thus, $\varphi$ blows up, by Proposition 2.4.

When $N \geqslant 3$ and $p=1+(4 / N)$, from Proposition 2.5, (4.8), $R(\lambda \varphi)=0$ and (2.3), we have

$$
\begin{aligned}
\frac{\mathrm{d}^{2}}{\mathrm{~d} t^{2}} \int|x|^{2}|\varphi|^{2} \mathrm{~d} x & \\
& =8 \int|\nabla \varphi|^{2} \mathrm{~d} x-8 \int\left[|x|^{2}|\varphi|^{2}+\frac{N(p-1)}{2(p+1)}|\varphi|^{p+1}\right] \mathrm{d} x \\
& <-8 \int\left[|x|^{2}|\varphi|^{2}+\frac{1}{1-\lambda^{2}}|\varphi|^{2}-\frac{2}{\left(1-\lambda^{2}\right)(p+1)}|\varphi|^{p+1}\right] \mathrm{d} x \\
& <-16 \int|x|^{2}|\varphi|^{2} \mathrm{~d} x+\frac{8}{\lambda^{p-1}\left(1-\lambda^{2}\right)}\left[-\lambda^{p-1}+1-\frac{\left(1-\lambda^{2}\right)(p-1) N}{4}\right] \int|\varphi|^{2} \mathrm{~d} x \\
& <\frac{8}{\lambda^{p-1}\left(1-\lambda^{2}\right)}\left[-\lambda^{p-1}+1-\frac{\left(1-\lambda^{2}\right)(p-1) N}{4}\right] \int|\varphi|^{2} \mathrm{~d} x \\
& =\frac{8}{\lambda^{p-1}\left(1-\lambda^{2}\right)}\left[-\lambda^{4 / N}+\lambda^{2}\right] \int|\varphi|^{2} \mathrm{~d} x \\
& =\frac{8}{\lambda^{p-1}\left(1-\lambda^{2}\right)}\left[-\lambda^{4 / N}+\lambda^{2}\right] \int\left|\varphi_{0}\right|^{2} \mathrm{~d} x .
\end{aligned}
$$

Set $f(\lambda)=-\lambda^{4 / N}+\lambda^{2}$, where $\lambda \in(0,1)$. When $N \geqslant 3$, we obtain $0<4 / N<2$. Therefore, $\lambda^{4 / N}>\lambda^{2}$, where $\lambda \in(0,1)$. Then $f(\lambda)<0$. Therefore, it follows that

$$
\frac{\mathrm{d}^{2}}{\mathrm{~d} t^{2}} \int|x|^{2}|\varphi|^{2} \mathrm{~d} x<-C<0
$$

which yields that the solution $\varphi$ of the Cauchy problem (1.1) blows up in a finite time in $H$.

Remark 4.3. When $N=1$, we unfortunately cannot find the sharp threshold between the global solutions and the blowing-up solutions of the Cauchy problem (1.1) for the critical power, $p=5$.

The following theorem answers the question: how small are the initial data, such that the Cauchy probelm (1.1) has global solutions for $p=1+(4 / N)$ ? 
Theorem 4.4. Let $p=1+(4 / N)$ and $N \geqslant 2$. If $\varphi_{0} \in H$ and satisfies

$$
\frac{1}{2}\left\|\varphi_{0}\right\|_{H}^{2}=\frac{1}{2} \int\left[\left|\nabla \varphi_{0}\right|^{2}+\left|\varphi_{0}\right|^{2}+|x|^{2}\left|\varphi_{0}\right|^{2}\right] \mathrm{d} x<d,
$$

then the solution $\varphi$ of (1.1) with the initial datum $\varphi_{0}$ exists globally on $t \in[0, \infty)$.

Proof. Let $\varphi_{0} \neq 0$ and let it satisfy (4.11). Then obviously $I\left(\varphi_{0}\right)<d$. Now we show that $\varphi_{0}$ also satisfies $R\left(\varphi_{0}\right)>0$. Firstly, we prove that

$$
R\left(\varphi_{0}\right)=\frac{1}{2} \int\left[\left|\varphi_{0}\right|^{2}-\frac{2}{p+1}\left|\varphi_{0}\right|^{p+1}\right] \mathrm{d} x \neq 0 .
$$

Otherwise, $\varphi_{0} \in \Sigma$. It follows that $I\left(\varphi_{0}\right)=R\left(\varphi_{0}\right)+S\left(\varphi_{0}\right)=S\left(\varphi_{0}\right)<d$, which is contradictory to Theorem 3.1. Therefore, if $R\left(\varphi_{0}\right)>0$ were not true, we would have

$$
R\left(\varphi_{0}\right)=\frac{1}{2} \int\left[\left|\varphi_{0}\right|^{2}-\frac{2}{p+1}\left|\varphi_{0}\right|^{p+1}\right] \mathrm{d} x<0 .
$$

Thus, there exists a $\mu \in(0,1)$ such that

$$
R\left(\mu \varphi_{0}\right)=\frac{1}{2} \int\left[\mu^{2}\left|\varphi_{0}\right|^{2}-\frac{2 \mu^{p+1}}{p+1}\left|\varphi_{0}\right|^{p+1}\right] \mathrm{d} x=0,
$$

which means that $\mu \varphi_{0} \in \Sigma$. But, by (4.11), we have

$$
\begin{aligned}
S\left(\mu \varphi_{0}\right) & =\frac{1}{2} \mu^{2} \int\left[\left|\nabla \varphi_{0}\right|^{2}+|x|^{2}\left|\varphi_{0}\right|^{2}\right] \mathrm{d} x \\
& <\frac{1}{2} \int\left[\left|\nabla \varphi_{0}\right|^{2}+|x|^{2}\left|\varphi_{0}\right|^{2}\right] \mathrm{d} x \\
& <\frac{1}{2} \int\left[\left|\nabla \varphi_{0}\right|^{2}+|x|^{2}\left|\varphi_{0}\right|^{2}+\left|\varphi_{0}\right|^{2}\right] \mathrm{d} x \\
& <d
\end{aligned}
$$

which contradicts Theorem 3.1. These contradictions yield $\varphi_{0} \in K_{+}$. By using Theorem 4.2, we find that the solution $\varphi$ of (1.1) with the initial datum $\varphi_{0}$ exists globally on $t \in[0, \infty)$.

Acknowledgements. The project is supported by the Scientific Research Found of Sichuan Provincial Education Department (Grant no. 2006A063).

\section{References}

1. H. Berestycki and T. Cazenave, Instabilité des états stationnaires dans les équations de Schrödinger et de Klein-Gordon non linéaires, C. R. Acad. Sci. Paris Sér. I 293 (1981), 489-492.

2. C. C. Bradley, C. A. Sackett and R. G. Hulet, Bose-Einstein condensation of lithium: observation of limited condensate number, Phys. Rev. Lett. 78 (1997), 985-989. 
3. R. CARLES, Remarks on the nonlinear Schrödinger equation with harmonic potential, Annls Inst. H. Poincaré 3 (2002), 757-772.

4. R. CARles, Critical nonlinear Schrödinger equations with and without harmonic potential, Math. Models Meth. Appl. Sci. 12 (2002), 1513-1523.

5. T. Cazenave, An introduction to nonlinear Schrödinger equations, Textos Metodos de Matematicos, Volume 26 (Universidade Federal do Rio de Janeiro, Rio de Janeiro, 1996).

6. T. Cazenave and P. L. Lions, Orbital stability of standing waves for some nonlinear Schrödinger equations, Commun. Math. Phys. 85 (1982), 549-561.

7. G. G. Chen And J. ZhANG, Sharp threshold of global existence for nonlinear GrossPitaevskii equation in $\mathbb{R}^{N}$, IMA J. Appl. Math. 71 (2006), 232-240.

8. F. Dalfovo, S. Giorgini, L. P. Pitaevskit and S. Stringari, Theory of Bose-Einstein condensation in trapped gases, Rev. Mod. Phys. 71 (1999), 463-512.

9. D. FujIWARA, Remarks on convergence of the Feynman path integrals, Duke Math. J. 47 (1980), 559-600.

10. R. Fukuizumi And M. Ohta, Stability of standing waves for nonlinear Schrödinger equations with potentials, Diff. Integ. Eqns 16 (2003), 111-128.

11. R. T. Glassey, On the blowing up of solutions to the Cauchy problem for nonlinear Schrödinger equations, J. Math. Phys. 18 (1977), 1794-1797.

12. M. Grillakis, J. Shatah and W. Strauss, Stability theory of solitary waves in the presence of symmetry, I, J. Funct. Analysis 74 (1987), 160-197.

13. Y. Kagan, A. E. Muryshev and G. V. Shlyapnikov, Collapse and Bose-Einstein condensation in a trapped Bose gas with negative scattering length, Phys. Rev. Lett. 81 (1998), 933-937.

14. J. L. Lebowitz, H. A. Rose And E. R. Speer, Statistical mechanics of the nonlinear Schrödinger equation, J. Statist. Phys. 50 (1988), 657-687.

15. H. A. LEvine, Instability and nonexistence of global solutions to nonlinear wave equations of the form $P u_{t t}=-A u-\mathcal{F}(u)$, Trans. Am. Math. Soc. 92 (1974), 1-21.

16. Y. G. OH, Cauchy problem and Ehrenfest's law of nonlinear Schrödinger equations with potentials, J. Diff. Eqns 81 (1989), 255-274.

17. L. E. Payne And D. H. Sattinger, Saddle points and instability of nonlinear hyperbolic equations, Israel J. Math. 22 (1975), 273-303.

18. W. A. Strauss, Nonlinear wave equations, CBMS Regional Conference Series in Mathematics, Volume 73 (American Mathematical Society, Providence, RI, 1989).

19. T. Tsurumi And M. WAdati, Collapses of wave functions in multidimensional nonlinear Schrödinger equations under harmonic potential, J. Phys. Soc. Jpn. 66 (1997), 3031-3034.

20. M. I. WEINSTEIN, Nonlinear Schrödinger equations and sharp interpolations estimates, Commun. Math. Phys. 87 (1983), 567-576.

21. K. Yajima, On fundamental solution of time dependent Schrödinger equations, Contemp. Math. 217 (1998), 49-68.

22. V. E. Zakharov, Collapse of Langmuir waves, Sov. Phys. JETP 23 (1966), 1025-1033.

23. J. ZhAng, Stability of attractive Bose-Einstein condensates. J. Statist. Phys. 101 (2000), 731-746.

24. J. ZHANG, Stability of standing waves for nonlinear Schrödinger equations with unbounded potentials, Z. Angew. Math. Phys. 51 (2000), 498-503.

25. J. ZHANG, Cross-constrained variational problem and nonlinear Schrödinger equation, in Foundations of Computational Mathematics, Proc. SMALEFEST 2000 (ed. F. Cucker and J. M. Rojas), pp. 457-469 (World Scientific, 2002). 